\title{
A Look at Cleaning Effectiveness in Automated Dissolution Systems
}

Gregory K. Webster*, Xi Shao, Kenneth A. Nelson, and Matthew A. Gragg

Analytical Research \& Development, AbbVie Inc., North Chicago, IL, USA.

e-mail: gregory.webster@abbvie.com

\section{ABSTRACT}

Cleaning in any Good Manufacturing Practice (GMP) laboratory is an important aspect of the analytical experiment. The laboratory must ensure the equipment does not contain residual active pharmaceutical ingredients (APIs) or impurities that may affect the outcome of any current or future experiments. While this is standard practice for GMP manufacturing operations, common laboratory equipment is often held to less stringent standards. The potential manhours lost due to investigations for extraneous peaks and contamination can be significant and cause delays in releasing product.

Potential compliance issues related to ineffective cleaning are particularly important for dissolution instrumentation. Our laboratory has modeled the challenges of cleaning automated dissolution systems using representative soluble and poorly soluble APIs. Poorly soluble drugs often entail the use of surfactants in the dissolution media which also have a potential carryover issue. Using a manufacturing cleaning validation based approach, the study discussion presented will address the cleaning effectiveness for both sample and media considerations.

KEYWORDS: Automated dissolution, dissolution bath cleaning, poorly soluble drugs, surfactant

\section{INTRODUCTION}

C leaning in any Good Manufacturing Practice (GMP) laboratory is an important aspect of the analytical experiment. The laboratory must ensure the equipment does not contain residual active pharmaceutical ingredients (APIs) or impurities that may affect the outcome of any current or future experiments $(1,2)$.

Common laboratory equipment is often held to less stringent standards than manufacturing equipment, and potential man hours lost due to investigations for extraneous peaks and contamination related to unclean equipment can be significant and cause delays in releasing product. For compliance, efficiency, and cost savings, effective cleaning investigations of critical laboratory equipment should be maintained. An example of critical laboratory equipment that can be affected by poor cleaning strategies is dissolution instrumentation. Dissolution instrumentation is critical for characterizing tablet performance (3). Failure to effectively clean the equipment can not only lead to erroneously high percentdissolved results, it can also introduce unexpected extraneous peak responses that require costly and timeconsuming laboratory investigations. The laboratory must ascertain if the extraneous response came from the equipment or the product prior to proposing a solution. Unless the issue is systematic, the investigation will be challenged by definitively assigning instrument contamination as opposed to the possibility of superpotent, unit-dosage form.

Automated dissolution systems have become effective tools for improving drug testing throughput (4-30). The cleaning of dissolution vessels is seemingly straightforward, yet we have found in our laboratory that the cleaning of transfer lines is just as important, and sometimes insufficient in systems routinely testing a variety of APIs, dosage forms, and strengths. The challenge of using unattended systems in dissolution is that issues in cleaning are often not discovered until after data have been generated. System suitability criteria to run blank media through the system prior to analysis to demonstrate the absence of line contamination is commonly used with on-line UV analysis. This blank control assessment can hinder the efficiency of automated systems. In the literature applications cited, only Mathieu

* Corresponding author. 
et al. suitably addressed the issue of cleaning (10). It is the authors' contention here that automated systems should be operated with cleaning investigations in mind. It is far better to add cleaning steps to the sample queue than to open investigations afterwards. To address this need, the challenge of cleaning automated dissolution systems in our laboratory was studied using representative soluble (biopharmaceutics classification system [BCS] class I and III) and poorly soluble and (BCS class II and IV) APIs (31).

\section{MATERIALS AND METHODS}

\section{Materials}

Except where noted, the chemicals used for this investigation were American Chemical Society (ACS) grade or better and purchased from MilliporeSigma (St. Louis, MO, USA). A sodium phosphate $(\mathrm{pH} \mathrm{6.8)}$ dissolution media was made from Dilut-it dissolution media concentrate from J.T. Baker/Avantor Performance Materials Holdings, S.A. (Gliwice, Poland). Sodium dodecyl sulfate (SDS) was Calbiochem OmniPur grade from EMD Millipore (Billerica, MA, USA). Itraconazole was purchased from Tokyo Chemical Industry (TCI) America Inc. (Portland, OR, USA). Labsan $230 \mathrm{C}$ citric acid cleaner was purchased from Sanitation Strategies LLC (Bend, OR, USA). Ritonavir was synthesized in house by AbbVie Inc. (North Chicago, IL, USA)

In-house swabbing procedures used cellulose support pads (AP10 024 00, MilliporeSigma, Burlington, MA, USA) for ritonavir, and Alpha sampling swabs (TX715, Texwipe, USA) for itraconazole and acetaminophen.

\section{Automated Dissolution System}

The automated dissolution system used for this investigation was an AT-70 from SOTAX (Westborough, MA, USA). The system was configured for United States Pharmacopoeia (USP) apparatus 2 paddle methods. The AT-70 was controlled by WinSOTAXplus Advanced Dissolution Software (version 2.57, SOTAX). The Xtend SAM sample manager (SOTAX) was directly coupled to an UltiMate 3000 high-performance liquid chromatography (HPLC) system (Thermo Fisher Scientific, Waltham, MA, USA) and controlled by Atlas Chromatography Data System (CDS) (version 9.00.00.10711, Thermo Fisher Scientific).

The dissolution methods used for this investigation were consistent with compendial procedures (32). The filters used for the dissolution samples were $0.45-\mu \mathrm{m}$ GxF polyvinylidene difluoride (PVDF) (Acrodisc, Pall Corp., USA) for acetaminophen, $0.45-\mu \mathrm{m}$ nylon for ritonavir (Pall Corp.), and 2.7- $\mu \mathrm{m}$ Whatman grade GF/D (GE Healthcare Life Sciences, USA) for itraconazole.

\section{SOTAX AT 70 Cleaning Cycle}

The AT-70 triggers the cleaning cycle immediately after the test run is completed. The cleaning cycle includes (1) removing filters from the filter station; (2) emptying vessels using the hemispheric valves on the bottom of the vessels; (3) removing sinkers, pellet cartridges, or undissolved dosage form simultaneously while the valves are opened; (4) removing baskets and collecting them in a cleaning reservoir; (5) power washing the inner vessel surface, shafts, and paddles using high speed rotation and pressurized deionized (DI) water; and (6) cleaning the circulation path using pressurized, DI water.

\section{Design Approach}

For our study, validated manufacturing cleaning methods were used for glass (vessel) and stainless steel (paddles). Swab recoveries were established for these surfaces using standard coupon recovery procedures (33). System transfer lines were investigated using rinse sampling (34).

\section{RESULTS AND DISCUSSION}

\section{Cleaning Targets}

Specific limits have not been established for dissolution apparatus as they are not directly used in the manufacturing of drug products. The industry has used cleaning limits based on $10 \mathrm{ppm}$ for finished drug manufacture, 50-100 ppm for API manufacture or dose, and maximum allowable carryover (MAC) calculations (35-38). The idea behind GMP cleaning limits can be used as a justifiable detection benchmark for dissolution instruments. The difference for analytical equipment is method integrity, not patient risk. Patient safety is not the concern for analytical test equipment cleaning investigations. The cleaning investigation for analytical instrumentation is used to minimize the risk of carry-over and impurities that may affect the next test run.

\section{Traditional Dissolution Cleaning Studies}

Traditionally, dissolution laboratories have simply executed the test method, performed their standard cleaning procedures and then verified the bath was clean by measuring the response from blank media. A response of less than $1 \%$ of the dose was a typical acceptance criterion (39). For a response of greater than or equal to $1 \%$, additional cleaning would be required.

\section{Less Than 1\% Response}

A target response of less than $1 \%$ is suitable only if the next application of the system is known ahead of time. The benchmark assumes the system will be used for the same method again. This is a poor assumption in research and development (R\&D) laboratories. R\&D laboratories routinely schedule testing queues on their 
dissolution equipment that comprise differing APIs as well as differing doses. Automated dissolution systems return their maximum return on investment (ROI) when executing a series of unattended methods. These systems require flexibility in sequencing differing drugs and dose levels. Thus, a $1 \%$ target of a $100-\mathrm{mg}$ dose could pose a significant issue if the subsequent sample tested is of lower dosage strength (e.g., it becomes a $10 \%$ response of a $10-\mathrm{mg}$ dose and $100 \%$ response for a 1-mg dose). Each laboratory should assess their cleaning need based on the potency of the drug products used.

\section{Proposed Cleaning Target}

Generally, a 1-mg dose is a representative lower objective for tablet strength in development. A $1 \%$ target of a 1 -mg dose would be equivalent to a $0.1 \%$ response of a $10-\mathrm{mg}$ dose and a $0.01 \%$ response for a $100-\mathrm{mg}$ dose. Thus, targeting a $0.01 \%$ criterion for cleaning our test 100 -mg study samples is a practical, universal goal to use to minimize method integrity issues for automated dissolution applications. To make this target more discriminating, the cleaning procedure should ensure a $0.01 \%$ target when using a $100-\mathrm{mg}$ dose of a poorly soluble drug. Such challenges would present a worstcase scenario and justify an effective standard cleaning procedure to be used for all methods going forward on the automated system.

Although this assumption works well for a homogeneously dispersed residue on a vessel surface using a coupon approach, one should expect a heterogeneous contamination in transfer lines where threaded junctions may have build-up. Such a scenario would not be expected with homogeneous samples. Although it might be $1 \%$ of the 1-mg dose, such contamination is more likely to be detected in an early sampling timepoint. For a $100 \mathrm{mg}$ drug that has only released $9 \mathrm{mg}$ into $900 \mathrm{~mL}$ of medium, and when flowing that $0.01 \mathrm{mg} / \mathrm{mL}$ solution through a transfer line, such a heterogeneous contaminant is often found in the first passage of sample and leads to the suspect result we are trying to avoid. Such a scenario is never totally avoidable in any dissolution system. Our goal is to minimize its occurrence.

\section{Automated Dissolution Cleaning}

Automated dissolution equipment allows unattended removal of sinkers, pellet cartridges, and undissolved dosage form components at the completion of the run. The instrumentation then can apply vessel emptying and cleaning procedures. For the instrument used in this investigation, a series of 14 rotating power washers were employed by programming of cleaning procedures for vessels and tubes. Pressurized cold and hot DI water can be used for vessel cleaning and rinsing dissolution media from the transfer lines. As illustrated in Figure 1, swab investigations for the dissolution vessels were executed by outlining the dimension of a standard cleaning coupon on the outside glass of each vessel and them swabbing the glass inside within these markings.

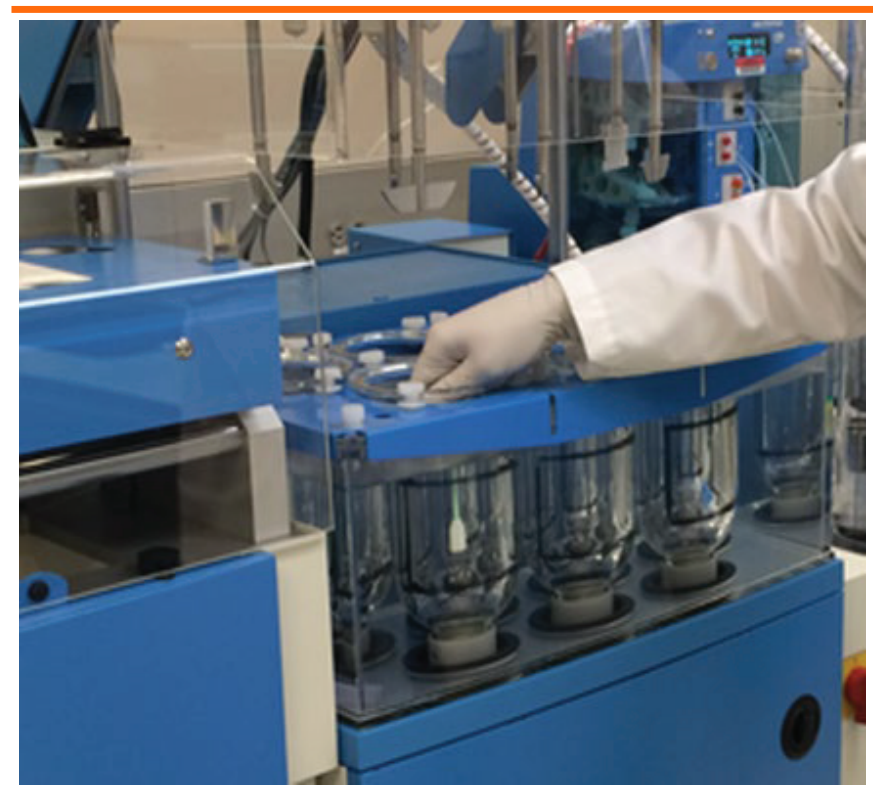

Figure 1. Illustration of swabbing the designated area dimension on glass dissolution vessels.

\section{Target Drugs}

For our investigations, acetaminophen was used to represent BCS class I and III drugs, ritonavir to represent a poorly soluble (BCS class IV) drug, and itraconazole to represent a known and challenging BCS class II drug. $\mathrm{HPLC}$ methods with practical detection limits in the range of $2-30 \mu \mathrm{g} / 100 \mathrm{~cm}^{3}$ were used to quantitate the media samples.

\section{Cleaning Study Methods}

Preliminary studies indicated that even for BCS class I and III drugs, a simple post run cleaning procedure, as designed by the manufacturer, left drug residue at levels higher than our $0.01 \%$ criteria, or $1.1 \mu \mathrm{g} / \mathrm{mL}$, in the media tested for $100-\mathrm{mg}$ doses. To address this, an extra cleaning run was added to the end of the sample queue to rinse the bath, paddle, and transfer lines with additional media prior to the DI water cleaning cycles. In addition, two cleaning cycles were used after the media blanks. To challenge the effectiveness of this revised procedure, 100-mg doses of each study drug were run through their validated dissolution method with surface exposures ranging from $30 \mathrm{~min}$ to $4 \mathrm{~h}$, based on the method routinely used for the tableted product. Next, the media blank was tested 
using 10-, 15- and 20-min hold times prior to draining the media. To check the glass and paddle surfaces, swab studies were run. To challenge the cleaning of the transfer lines, a blank run was executed after swabbing to test the rinsed lines. The revised cleaning procedure used for this investigation is listed in Table 1.

\section{Cleaning Study Results for Acetaminophen, Ritonavir, and Itraconazole}

The results for acetaminophen and ritonavir are listed in Tables 2 and 3, respectively. Acetaminophen, being highly soluble, showed efficient cleaning as expected. In addition, ritonavir, as a poorly soluble drug, also showed efficient cleaning with the procedures used. The method surfactants functioned to keep the drug soluble enough to be rinsed out of the system. The concern remains: at what point does surfactant residue itself becomes an issue? Future work will explore this impact upon automated systems. For now, the authors propose that investigators use the minimum amount of surfactant needed in their dissolution methods. To date, our procedure has been suitable for media containing up to $1 \%$ SDS. The preliminary results for itraconazole, which is a poorly soluble drug using low-acid $\mathrm{pH}$ media rather than a surfactant media, were higher than desired (Table 4). Additional studies into cleaning methods for itraconazole and media ensued (Table 5).

Each cleaning investigation must assess the chemistry, solubility, and solution stability issues that a drug may present. Itraconazole is a challenging drug to clean due to its poor aqueous solubility. Considering this challenge, the levels reported in Tables 4 and 5 may be suitable. To ensure the proposed $0.01 \%$ target could be obtained with this drug, further testing was initiated. Because of itraconazole's solubility in acidic media, end of sequence media rinses with either $0.1 \mathrm{~N} \mathrm{HCl}$ or acidic detergent (Labsan 230C) were tested (32). As with other BCS class II and IV drugs, cleaning improved in the presence of surfactant media.

\section{Optimized Cleaning Method for Automated Dissolution}

Given the vigorous vessel rinsing and the swabbing techniques employed in the swabbing studies, the data supports that carry-over issues, if present, are more likely to originate in the transfer lines than the dissolution vessel and paddle surfaces. In addition, BCS class II and IV compounds, when using surfactant media, did not pose a challenge to our cleaning investigation This was attributed to the solubilizing and rinsability of the surfactants used. With these drugs being more soluble in surfactant media, and because surfactant media residues are easier to liberate from material surfaces than simple aqueous media, there is less need to liberate these drugs from contact surfaces. In fact, our investigation showed that BCS class II and IV drugs in surfactant media can be rinsed away more easily than BCS class I and III drugs in simple aqueous media. Simple aqueous media does not inhibit the attraction of BCS class I and III drugs to surfaces. Except for itraconazole, the optimized cleaning sequence used at the end of the sample queue was effective at ensuring that the target of $0.01 \%$ of a $100-\mathrm{mg}$ dose was achieved. This procedure was also challenged with an additional proprietary BCS class I and III and BCS class II and IV drugs. While the results confirmed the procedure met the $0.01 \%$ cleaning target for a $100-\mathrm{mg}$ dose as well, the data is not presented here. Based on this investigation, the final optimized cleaning procedure only requires the Blank Media Method section of Table 1; i.e., four sample pulls totaling $400 \mathrm{~mL}$ of line flush and $16 \mathrm{~min}$ of flushing is reduced to only two sample pulls, totaling $200 \mathrm{~mL}$ of line flush and $8 \mathrm{~min}$ of flushing.

\section{Additional Recommendations for Automated Dissolution Runs}

The goal of this cleaning investigation was to minimize suspect result investigations. Two additional suggestions will assist in minimizing the time lost to examining suspect data. First, consider adding carryover standard spikes to methods when different drugs are being tested in the queue sequence. This enables the analyst to assign the identity and likely cause of a suspect peak that washed into the next method. Second, order sequence queues in the order of low to higher doses of the drug or drugs under investigations. This notion could also be expanded to cases where multiple actives are screened in an automated run. In those cases, the sequence should be optimized in order of weakest analytical signal (ultraviolet [UV] maxima) to strongest.

\section{CONCLUSION}

For the dissolution testing of BCS class I and III and BCS class II and IV drugs with surfactant media, suitable cleaning procedures have been developed that enable automated dissolution sequences with minimal risk of analyte carry-over. The cleaning benchmarks used were based on a $1 \%$ target for the lowest expected dose. This benchmark provided flexibility in the sequence queue. Cleaning procedures for the dissolution testing of BCS class II and IV drugs in the absence of surfactant media, such as itraconazole, should be investigated on an individual basis. 
Table 1. Study Cleaning Procedures for SOTAX AT-70 Automated Dissolution System

\begin{tabular}{|l|l|l|}
\hline Task & Procedure & Reasoning \\
\hline Media Flush Before Sampling & $100 \mathrm{~mL}$ & At $25 \mathrm{~mL} / \mathrm{min}$, this gives 4 min of line flush per pull \\
\hline Blank Media Method & 4 Sample Pulls & Totals $400 \mathrm{~mL}$ of line flush and 16 min of flushing \\
\hline Default Cleaning Method & 2 Cycles & Thorough rinsing of media with deionized water \\
\hline
\end{tabular}

Table 2. Acetaminophen Cleaning Study Results

\begin{tabular}{|c|c|c|c|c|c|c|c|c|}
\hline Vessel & $\begin{array}{c}\text { Media } \\
10 \text { Min }\end{array}$ & $\begin{array}{c}\text { Media } \\
\mathbf{1 5} \text { Min }\end{array}$ & $\begin{array}{c}\text { Swab Blank } \\
\text { (Paddles) }\end{array}$ & $\begin{array}{c}\text { Glass } \\
\text { Surface }\end{array}$ & $\begin{array}{c}\text { Blank Run } \\
\mathbf{5} \text { Min }\end{array}$ & $\begin{array}{c}\text { Blank Run } \\
\mathbf{1 0} \text { Min }\end{array}$ & $\begin{array}{c}\text { Blank Run } \\
\mathbf{1 5} \text { Min }\end{array}$ & $\begin{array}{c}\text { Blank Run } \\
\mathbf{2 0} \text { Min }\end{array}$ \\
\hline Blank & 0.002 & 0.028 & 0.000 & 0.000 & 0.000 & 0.000 & 0.000 & 0.000 \\
\hline Blank & 0.014 & 0.004 & 0.000 & 0.000 & 0.000 & 0.000 & 0.000 & 0.000 \\
\hline 1 & 0.030 & 0.017 & 0.000 & 0.000 & 0.000 & 0.000 & 0.000 & 0.000 \\
\hline 2 & 0.033 & 0.019 & 0.000 & 0.000 & 0.000 & 0.000 & 0.000 & 0.000 \\
\hline 3 & 0.029 & 0.022 & - & 0.000 & 0.000 & 0.000 & 0.000 & 0.000 \\
\hline 4 & 0.024 & 0.018 & - & 0.000 & 0.000 & 0.000 & 0.000 & 0.000 \\
\hline 5 & 0.029 & 0.021 & - & 0.000 & 0.000 & 0.000 & 0.000 & 0.000 \\
\hline 6 & 0.026 & 0.020 & - & 0.000 & 0.000 & 0.000 & 0.000 & 0.000 \\
\hline Average & $\mathbf{0 . 0 2 8}$ & $\mathbf{0 . 0 1 9}$ & $\mathbf{0 . 0 0 0}$ & $\mathbf{0 . 0 0 0}$ & $\mathbf{0 . 0 0 0}$ & $\mathbf{0 . 0 0 0}$ & $\mathbf{0 . 0 0 0}$ & $\mathbf{0 . 0 0 0}$ \\
\hline
\end{tabular}

Results adjusted to represent the impact (in $\mu \mathrm{g} / \mathrm{mL}$ ) to the next dissolution run

Table 3. Ritonavir Cleaning Study Results

\begin{tabular}{|c|c|c|c|c|c|c|c|c|c|c|}
\hline Vessel & $\begin{array}{c}\text { Media } \\
10 \text { Min }\end{array}$ & $\begin{array}{c}\text { Media } \\
\mathbf{1 5} \text { Min }\end{array}$ & $\begin{array}{c}\text { Media } \\
\mathbf{2 0} \text { Min }\end{array}$ & $\begin{array}{c}\text { Media } \\
\mathbf{2 5} \text { Min }\end{array}$ & Paddles & $\begin{array}{c}\text { Glass } \\
\text { Surface }\end{array}$ & $\begin{array}{c}\text { Blank Run } \\
\mathbf{5} \text { Min }\end{array}$ & $\begin{array}{c}\text { Blank Run } \\
10 \text { Min }\end{array}$ & $\begin{array}{c}\text { Blank Run } \\
15 \text { Min }\end{array}$ & $\begin{array}{c}\text { Blank Run } \\
\mathbf{2 0} \text { Min }\end{array}$ \\
\hline Blank & 0.000 & 0.000 & 0.000 & 0.000 & 0.000 & 0.000 & 0.000 & 0.000 & 0.000 & 0.000 \\
\hline 1 & 0.093 & 0.071 & 0.072 & 0.068 & 0.001 & 0.000 & 0.000 & 0.000 & 0.000 & 0.000 \\
\hline 2 & 0.087 & 0.061 & 0.057 & 0.059 & 0.000 & 0.000 & 0.000 & 0.000 & 0.000 & 0.000 \\
\hline 3 & 0.096 & 0.077 & 0.069 & 0.068 & - & 0.000 & 0.000 & 0.000 & 0.000 & 0.000 \\
\hline 4 & 0.260 & 0.292 & 0.309 & 0.310 & - & 0.000 & 0.000 & 0.000 & 0.000 & 0.000 \\
\hline 5 & 0.103 & 0.072 & 0.068 & 0.067 & - & 0.000 & 0.000 & 0.000 & 0.000 & 0.000 \\
\hline 6 & 0.101 & 0.063 & 0.060 & 0.059 & - & 0.000 & 0.000 & 0.000 & 0.000 & 0.000 \\
\hline Average & $\mathbf{0 . 1 2 3}$ & $\mathbf{0 . 1 0 6}$ & $\mathbf{0 . 1 0 6}$ & $\mathbf{0 . 1 0 5}$ & $\mathbf{0 . 0 0 0}$ & $\mathbf{0 . 0 0 0}$ & $\mathbf{0 . 0 0 0}$ & $\mathbf{0 . 0 0 0}$ & $\mathbf{0 . 0 0 0}$ & $\mathbf{0 . 0 0 0}$ \\
\hline
\end{tabular}

Results adjusted to represent the impact (in $\mu \mathrm{g} / \mathrm{mL}$ ) to the next dissolution run

Table 4. Itraconazole Cleaning Study Results for Transfer Lines

\begin{tabular}{|c|c|c|c|c|c|c|}
\hline \multirow{2}{*}{ Vessel } & \multicolumn{6}{|c|}{ Cleaning Procedure } \\
\hline & A & B & C & D & E & $F$ \\
\hline $\mathrm{T} 1$ & 0.40 & 0.10 & 0.04 & 0.03 & 0.05 & 0.00 \\
\hline $\mathrm{T} 2$ & 0.20 & 0.04 & 0.02 & 0.01 & 0.01 & 0.00 \\
\hline T3 & 0.40 & 0.13 & 0.06 & 0.03 & 0.02 & 0.00 \\
\hline T4 & 0.20 & 0.06 & 0.03 & 0.01 & 0.01 & 0.00 \\
\hline T5 & 0.20 & 0.06 & 0.02 & 0.01 & 0.02 & 0.01 \\
\hline T6 & 0.20 & 0.04 & 0.02 & 0.00 & 0.01 & 0.00 \\
\hline Average & 0.27 & 0.07 & 0.03 & 0.02 & 0.02 & 0.00 \\
\hline
\end{tabular}

Data expressed as \% of a $100 \mathrm{mg}$ itraconazole

A: Itraconazole with one cleaning program

$B$ : Itraconazole with two 15-min infinity runs of $0.1 \mathrm{~N} \mathrm{HCl}$ and five cleaning programs

C: Itraconazole with two 50 -min infinity runs of $0.1 \mathrm{~N} \mathrm{HCl}$ and five cleaning programs

D: Itraconazole with two 50 -min infinity runs $(0.1 \mathrm{~N} \mathrm{HCl}$ followed by $20 \%$ Labsan $230 \mathrm{C})$ and five cleaning programs

E: Itraconazole with two 50-min infinity runs $(0.1 \mathrm{~N} \mathrm{HCl} \mathrm{followed} \mathrm{by} 40 \%$ Labsan 230C) and five cleaning programs

F: Itraconazole with two 50 -min infinity runs ( $0.1 \mathrm{~N} \mathrm{HCl}$ followed by $2.3 \%$ SDS) and five cleaning programs 
Table 5. Itraconazole Cleaning Study Results for Glass and Paddle Surfaces

\begin{tabular}{|c|c|c|c|c|c|c|}
\hline \multirow{2}{*}{ Vessel } & \multicolumn{6}{|c|}{ Cleaning Procedure } \\
\hline & A & B & C & D & E & $\mathbf{F}$ \\
\hline Glass 1 & 4.30 & 0.14 & 0.07 & 0.12 & 0.02 & 0.02 \\
\hline Glass 2 & 1.60 & 0.08 & 0.29 & 0.06 & 1.07 & 0.02 \\
\hline Glass 3 & 6.80 & 0.09 & 0.25 & 0.05 & 0.02 & 0.04 \\
\hline Glass 4 & 5.20 & 0.10 & 0.11 & 0.05 & 0.01 & 0.03 \\
\hline Glass 5 & 0.70 & 0.00 & 29.70 & 0.13 & 1.52 & 0.04 \\
\hline Glass 6 & 0.80 & 0.53 & 0.04 & 0.08 & 0.02 & 0.04 \\
\hline Paddle & 17.60 & 0.46 & 0.09 & 0.35 & 0.05 & 0.06 \\
\hline Average & 5.29 & 0.20 & 4.36 & 0.12 & 0.39 & 0.04 \\
\hline
\end{tabular}

Data expressed as \% of $100 \mathrm{mg}$ itraconazole

A: Itraconazole with one cleaning program

B: Itraconazole with two 15-min infinity runs of $0.1 \mathrm{~N} \mathrm{HCl}$ and five cleaning programs

C: Itraconazole with two 50-min infinity runs of $0.1 \mathrm{~N} \mathrm{HCl}$ and five cleaning programs

D: Itraconazole with two 50 -min infinity runs $(0.1 \mathrm{~N} \mathrm{HCl}$ followed by $20 \%$ Labsan 230C) and five cleaning programs

E: Itraconazole with two 50 -min infinity runs $(0.1 \mathrm{~N} \mathrm{HCl} \mathrm{followed} \mathrm{by} 40 \%$ Labsan $230 \mathrm{C})$ and five cleaning programs

F: Itraconazole with two 50 -min infinity runs $(0.1 \mathrm{~N} \mathrm{HCl}$ followed by $2.3 \%$ SDS) and five cleaning programs

The data not only confirm that surfactants in dissolution media help with cleaning residual components from the system but from our experience in using automated systems has us observing that it is easier to clean for BCS II/IV drug methods when using surfactants than experienced with traditional BCS class I and III drug method investigations. This again is attributed to the use of surfactants in the media. Finally, laboratories should not assume that instrumentation used with BCS I/III drug methods are easily cleansed using minimal default methods. Investigators should note that BCS I/III drugs can have affinity for transfer lines as well.

\section{ACKNOWLEDGMENT}

This work was presented in part at the 2017 PITTCON Conference \& Expo, Chicago, IL, USA.

\section{CONFLICT OF INTEREST}

The authors are employees of AbbVie and may own AbbVie stock. AbbVie sponsored and funded the study, contributed to the design, participated in the collection, analysis, and interpretation of data, and in writing, reviewing, and approval of the final publication.

\section{REFERENCES}

1. Ghosh, A.; Dey, S. Overview of cleaning validation in pharmaceutical industry. IJPQA. 2010, 2, 26-30.

2. LeBlanc, D. A. Validated Cleaning Technologies For Pharmaceutical Manufacturing; CRC Press: Boca Raton, 2000.

3. Webster, G. K.; Bell, R. G.; Jackson, J. D. Poorly Soluble Drugs: Dissolution and Drug Release (Pan Stanford Series on Pharmaceutical Analysis; Pan Stanford Series on Pharmaceutical Analysis. Pan Stanford Publishing: Singapore, 2016.
4. Neitring, I.; Yapchanyk, E. Fully automated dissolution and drug release testing. Am. Lab. 1987, 19, 62.

5. George, R. C.; Cornelius, K. E.; Contario, J. J. Automated dissolution testing of sustained-release tablets. Am. Lab. 1988, 20, 106-112.

6. Paul, A. M.; Good, K. S.; Tucker, K. A.; Strimaitis, J. R. Automated dissolution testing using a laboratory robotic system. Am. Lab. 1989, 21, 80-91.

7. Aronson, B.; Faber, P.; Raya, M. Comparison of dissolution rate release profiles using an automated dissolution/HPLC system. Am. Lab. 1989, 8, 17-20.

8. Simonian, M. H.; Anderson, J. C. Automated dissolution testing utilizing UV-visible spectrophotometers. Am. Lab. 1991, 23 , 2.

9. Dunkle, J. D.; Gleason, D. J.; Hokanson, G. C. A flexible automated dissolution testing system for use with either USP apparatus 1 or USP apparatus 2. Pharm. Res. 1992, 9, 578-581.

10. Mathieu, R. J.; Van, O.; Nicholson, D. G. M.; Kanfer, I. Automated dissolution testing using on-line low-dispersion HPLC. Am. Lab. 1992, 24, 24.

11. Wood, J. A.; Goras, J. T. Validating an automated dissolution method for a tablet containing both sustained and immediate release active components. J. Pharm. Biomed. Anal. 1993, 11, 1361-1365. DOI: 10.1016/0731-7085(93)80127-M.

12. Li, J. B.; Rahn, P. C. Automated dissolution testing of topical drug formulations using franz cells and HPLC analysis. Pharm. Technol. 1993, 17, 54.

13. Fuerte, V.; Maldonado, M. The multicomponent automated dissolution system: an alternative in the development and pharmaceutical analysis of generic polydrugs. Dissolution Technol. 1999, 6, 12-16. DOI: 10.14227/DT060299P12.

14. Fuerte, V.; Maldonado, M.; Rees, G. D. The multicomponent automated dissolution system: an alternative in the 
development and pharmaceutical analysis of generic polydrugs. J. Pharm. Biomed. Anal. 1999, 21, 267-272. DOI: 10.1016/ S0731-7085(99)00122-3.

15. Rogers, P.; Hailey, P. A.; Johnson, G. A.; Dight, V. A.; Read, C.; Shingler, A.; Savage, P.; Roche, T.; Mondry, J. A comprehensive and flexible approach to the automated-dissolution testing of pharmaceutical drug products incorporating direct UV-Vis fiberoptic analysis, on-line fluorescence analysis, and off-line storage options. Lab. Robpt. Autom. 2000, 12, 12-22. DOI: 10.1002/ (sici)1098-2728(2000)12:1<12::aid-Ira3>3.0.co;2-f.

16. Steinman, T. A. Qualification of a $Z_{y m a r k}{ }^{\circledR}$ Multidose $^{\circledR}$ automated dissolution workstation for dissolution testing of fexofenadine-HCl capsules. JALA. 2000, 5, 81-86. DOI: 10.1016/ s1535-5535(04)00105-4.

17. Li, B.; Zhang, Z.; Liu, W. Flow-injection system for automated dissolution testing of isoniazid tablets with chemiluminescence detection. Talanta. 2001, 54, 697-702. DOI: 10.1016/S00399140(01)00325-3.

18. Steinman, T. A.; Parente, E. Qualification of a Zymark ${ }^{\circledR}$ MultiDose $^{\circledR}$ automated dissolution workstation for dissolution testing of fexofenadine $\mathrm{HCl}$ capsules. Dissolution Technol. 2001, 8, 1-7. DOI: 10.14227/DT080201P12.

19. Sadowitz, J. P. Expediting the formulation development process with the aid of automated dissolution in analytical research and development. J. Autom. Methods Manag. Chem. 2001, 23, 173177. DOI: 10.1155/S1463924601000219.

20. Zhao, L.; Li, B.; Zhang, Z.; Lin, J. Chemiluminescent flow-through sensor for automated dissolution testing of analgin tablets using manganese dioxide as oxidate. Sens. Actuators, B Chem. 2004, 97, 266-271. DOI: 10.1016/j.snb.2003.08.026.

21. Liu, X.; Cai, F. Flow-injection system for automated dissolution testing of chlorpromazine hydrochloride tablets with chemiluminescence detection. Xinan Minzu Daxue Xuebao. Ziran Kexue Ban 2004, 30, 286-290.

22. Zou, X.; Jin, W.; Zhou, J.; Cao, Y.; Liu, G.; Zhang, L.; Jin, Q. A novel automated dissolution test analyzer for composite tablets dissolution test [in Chinese]. Fenxi Huaxue 2006, 34, S303-S305.

23. Kozlowski, B.; John, C. Specialty automation groups and implementation of automated dissolution in early development settings. Am. Pharm. Rev. 2008, 11, 53-55.

24. Lo, L.; Lu, X.; Lloyd, D. Dissolution testing of a controlled-release capsule formulation: challenges and solutions using a semiautomated dissolution system. Dissolution Technol. 2013, 20, 6-12. DOI: 10.14227/DT200213P6.

25. Ibrahim, S. A.; Martini, L. Automated dissolution for entericcoated aspirin tablets: a case study for method transfer to a RoboDis II. J. Lab. Autom. 2014, 19, 375-380. DOI: 10.1177/2211068213520401.

26. Romberger, G.; Hein, E.; Do, M.; Lavrich, D.; Gallagher, K.; Kozlowski, B. Content uniformity testing through utilization of automated dissolution technologies. Am. Pharm. Rev. 2014, 17, 67-69.

27. Petruševska, M.; Horvat, M.; Peternel, L.; Kristan, K. Highthroughput automated dissolution method applicable for a wide dose range of controlled release pellets. Drug Dev. Ind. Pharm. 2016, 42, 1149-1157. DOI: 10.3109/03639045.2015.1117485.

28. Hai-wei, H.; Song, Y.; Li-ju, Y.; Lan, H.; Qi-ming, Z.; Bao-ming, N.; Wilkinson, K.; Shi, W.; Li-fang, S. New innovations in testing sustained-release tablets using an automated dissolution system with online dilution. Dissolution Technol. 2015, 22, 13-16. DOI: 10.14227/DT220115P13.

29. Shah, K. P.; Chang, M.; Riley, C. M. Automated analytical systems for drug development studies. II - A system for dissolution testing. J. Pharm. Biomed. Anal. 1994, 12, 1519-1527. DOI: 10.1016/0731-7085(94)00103-0.

30. Shah, K. P.; Chang, M.; Riley, C. M. Automated analytical systems for drug development studies 3. Multivessel dissolution testing system based on microdialysis sampling. J. Pharm. Biomed. Anal. 1995, 13, 1235-1241. DOI: 10.1016/0731-7085(95)01529-T.

31. Amidon, G. L.; Lennernäs, H.; Shah, V. P.; Crison, J. R. A theoretical basis for a biopharmaceutic drug classification: the correlation of in vitro drug product dissolution and in vivo bioavailability. Pharm. Res. 1995, 12, 413-420. DOI: 10.1023/A:1016212804288.

32. Dissolution Method Database. U.S. Food and Drug Administration website. Updated Oct 2019. https://www.accessdata.fda.gov/ scripts/cder/dissolution/ (accessed May 1, 2020).

33. Yang, P.; Burson, K.; Feder, D.; Macdonald, F. Method development of swab sampling for cleaning validation of a residual active pharmaceutical ingredient. Pharm. Technol. 2005, 29, 84-94.

34. Le Blanc, D. A. Rinse Sampling for cleaning validation studies. Pharm. Technol. 1998, 22, 66-74.

35. LeBlanc, D. A. Establishing scientifically justified acceptance criteria for the cleaning validation of APIs. Pharm. Technol. 2000, 24, 160-168.

36. Fourman, G. L.; Mullen, M. V. Cleaning validation acceptance limits for pharmaceutical manufacturing operations. Pharm. Technol. 1993, 17, 54-60.

37. Crevoisier, M.; Lovsin Barle, E.; Flueckiger, A.; Dolan, D. G.; Ader, A.; Walsh, A. Cleaning limits-why the 10-ppm criterion should be abandoned. Pharm. Technol. 2016, 40, 52-56.

38. Walsh, A. Cleaning validation for the 21st century: acceptance limits for active pharmaceutical ingredients (APIs): Part I. Pharm. Engineering. 2011, 31, 74-83.

39. <1092> The Dissolution Procedure: Development and Validation. In United States Pharmacopeia and National Formulary (USP 42-NF 37). The United States Pharmacopeial Convention, Inc.: Rockville, MD, 2019. 